\title{
Incidence and recurrence of new-onset atrial fibrillation detected during hospitalization for non-cardiac surgery: a systematic review and meta-analysis
}

\section{Incidence et récurrence de nouvelle fibrillation auriculaire détectée pendant l'hospitalisation pour une chirurgie non cardiaque : revue systématique et méta-analyse}

William F. McIntyre, MD FRCPC (D) - Maria E. Vadakken, MBBS • Anand S. Rai, BSc •

Terry Thach, MD • Wajahat Syed, MD • Kevin J. Um, MD • Omar Ibrahim, MD • Shreyash Dalmia, MD • Akash Bhatnagar, MD • Pablo A. Mendoza, BSc • Alexander P. Benz, MD • Shrikant I. Bangdiwala, PhD • Jessica Spence, MD FRCPC • Graham R. McClure, MD • Jessica T. Huynh, MD • Tianyi Zhang • Toru Inami, MD PhD • David Conen, MD MPH • P. J. Devereaux, MD PhD FRCPC • Richard P. Whitlock, MD PhD FRCSC · Jeff S. Healey, MD FRCPC • Emilie P. Belley-Côté, MD PhD FRCPC

Received: 21 October 2020/Revised: 11 January 2021 / Accepted: 11 January 2021 / Published online: 23 February 2021

(C) Canadian Anesthesiologists' Society 2021

\begin{abstract}
Purpose This systematic review aimed to summarize reports of the incidence and long-term recurrence of new-onset atrial fibrillation (AF) associated with noncardiac surgery.

Sources We searched CENTRAL, MEDLINE and EMBASE
\end{abstract}

Supplementary Information The online version of this article (https://doi.org/10.1007/s12630-021-01944-0) contains supplementary material, which is available to authorized users.

W. F. McIntyre, MD FRCPC ( $)$ - J. Spence, MD FRCPC . D. Conen, MD MPH - P. J. Devereaux, MD PhD FRCPC .

J. S. Healey, MD FRCPC

Population Health Research Institute, McMaster University,

Hamilton, ON, Canada

e-mail: William.McIntyre@phri.ca

Department of Medicine, McMaster University, Hamilton, ON, Canada

Department of Health Research Methods, Evidence, and Impact, McMaster University, Hamilton, ON, Canada

M. E. Vadakken, MBBS - A. S. Rai, BSc · A. Bhatnagar, MD · T. Zhang

Population Health Research Institute, McMaster University,

Hamilton, ON, Canada

T. Thach, MD $\cdot$ W. Syed, MD

Faculty of Health Sciences, McMaster University, Hamilton,

ON, Canada from inception to November 2019. We included studies that reported on the incidence of new-onset perioperative $A F$ during hospitalization for non-cardiac surgery and/or AF recurrence in such patients following discharge. Reviewers screened articles and abstracted data independently and in duplicate. We assessed study quality by appraising methodology for collecting AF history, incident AF during hospitalization, and AF recurrence after discharge.

K. J. Um, MD · O. Ibrahim, MD · S. Dalmia, MD .

J. T. Huynh, MD

Department of Medicine, McMaster University, Hamilton, ON, Canada

P. A. Mendoza, BSc

Department of Health Research Methods, Evidence, and Impact, McMaster University, Hamilton, ON, Canada
A. P. Benz, MD · S. I. Bangdiwala, PhD
Population Health Research Institute, McMaster University, Hamilton, ON, Canada
Department of Health Research Methods, Evidence, and Impact, McMaster University, Hamilton, ON, Canada
G. R. McClure, MD
Department of Surgery, McMaster University, Hamilton, ON, Canada 
Principal findings From 39,233 citations screened, 346 studies that enrolled a total of 5,829,758 patients met eligibility criteria. Only 27 studies used prospective, continuous inpatient electrocardiographic (ECG) monitoring to detect incident AF. Overall, the incidence of postoperative $A F$ during hospitalization ranged from 0.004 to $50.3 \%$, with a median [interquartile range] of 8.7 [3.8-15.0]\%. Atrial fibrillation incidence varied with type of surgery. Prospective studies using continuous ECG monitoring reported significantly higher incidences of $A F$ than those that did not $(13.9 \%$ vs $1.9 \%$, respectively; $P<$ 0.001). A total of 13 studies (25,726 patients) with followup up to 5.4 years reported on AF recurrence following hospital discharge; only one study used a prospective systematic monitoring protocol. Recurrence rates ranged from 0 to $37.3 \%$.

Conclusions Rates of AF incidence first detected following non-cardiac surgery and long-term $A F$ recurrence vary markedly. Differences in the intensity of ECG monitoring and type of surgery may account for this variation.

Trial registration PROSPERO (CRD42017068055); registered 1 September 2017.

\section{Résumé}

Objectif Cette revue systématique visait à résumer les comptes rendus sur l'incidence et la récurrence à long terme de la fibrillation auriculaire (FA) de novo associée à une chirurgie non cardiaque.

Sources Nous avons effectué des recherches dans les bases de données CENTRAL, MEDLINE et EMBASE de leur création à novembre 2019. Nous avons inclus les études ayant examiné l'incidence de nouvelle FA périopératoire pendant l'hospitalisation pour une chirurgie non cardiaque et/ou la récurrence de la FA

T. Inami, MD PhD

Department of Cardiovascular Medicine, Nippon Medical

School, Tokyo, Japan

R. P. Whitlock, MD PhD FRCSC

Population Health Research Institute, McMaster University,

Hamilton, ON, Canada

Department of Medicine, McMaster University, Hamilton, ON, Canada

Department of Surgery, McMaster University, Hamilton, ON, Canada

E. P. Belley-Côté, MD PhD FRCPC

Population Health Research Institute, McMaster University, Hamilton, ON, Canada

Department of Medicine, McMaster University, Hamilton, ON, Canada chez de tels patients après leur congé. Les chercheurs ont passé en revue les articles et les données extraites de manière indépendante et en double. Nous avons estimé la qualité des études en évaluant la méthodologie de collecte des antécédents de FA, de l'incident de FA pendant l'hospitalisation et de la récurrence de FA après le congé. Constatations principales Sur les 39233 citations examinées, 346 études portant sur un total de 5829758 patients ont répondu à nos critères d'admissibilité. Seulement 27 études ont utilisé un monitorage electrocardiographique (ECG) continu prospectif et des patients hospitalisés pour détecter les incidents de FA. Dans l'ensemble, l'incidence de FA postopératoire pendant l'hospitalisation allait de 0,004 à $50,3 \%$, avec une médiane [écart interquartile] de 8,7 [3,8-15,0] \%. L'incidence de fibrillation auriculaire variait en fonction du type de chirurgie. Des études prospectives utilisant un monitorage continu par ECG ont fait état d'incidences significativement plus elevées de FA que celles sans monitorage continu $(13,9 \%$ vs $1,9 \%$, respectivement; $P<0,001)$. Au total, 13 études (25 726 patients) avec un suivi allant jusqu'à 5,4 ans ont rapportéleurs données sur la récurrence de FA après le congé de l'hôpital; seule une étude a utilisé un protocole de monitorage prospectif systématique. Les taux de récurrence allaient de 0 à $37,3 \%$. Conclusion Les taux d'incidence de nouvelle FA détectés après une chirurgie non cardiaque et la récurrence à long terme de FA varient considérablement. Les différences du degré de monitorage par ECG et le type de chirurgie pourraient expliquer cette variation.

Enregistrement de l'étude PROSPERO (CRD42017068055); enregistrée le ler septembre 2017.

Keywords atrial fibrillation $\cdot$ secondary $\cdot$ reversible stroke $\cdot$ perioperative $\cdot$ telemetry

Atrial fibrillation (AF) is the most common serious heart rhythm disorder and is frequently detected for the first time after surgery. ${ }^{1-3}$ Published articles report a wide range in the incidence of new-onset perioperative $\mathrm{AF}^{2,4}$ which may also vary according to the type of surgery, the baseline risk in the population under study, the approach to electrocardiography (ECG) monitoring, and the definition used for perioperative $\mathrm{AF}$ (e.g., $\geq 30$ seconds $\mathrm{AF}$ duration, $\mathrm{AF}$ detected on a monitor, or AF that results in symptoms or requires treatment). ${ }^{2,4}$ New-onset perioperative AF has been associated with higher rates of adverse outcomes and mortality as well as longer length of hospital stay after noncardiac surgery. ${ }^{5-7}$ In the perioperative setting, AF often resolves before hospital discharge, and the long-term prognosis of patients with $\mathrm{AF}$ occurring transiently with 
stress (AFOTS) is not well-defined..$^{2,8-10}$ The occurrence of AFOTS could be secondary to physiologic stressors associated with surgery with no long-term consequences; but it may also be newly detected paroxysmal $\mathrm{AF}^{2}$ Understanding whether AFOTS is a short-term problem or the first presentation of a chronic disease (i.e., paroxysmal AF) is critical because the risk of ischemic stroke associated with paroxysmal AF can be reduced with oral anticoagulants (OAC). ${ }^{2}$ Clinical practice guidelines and position statements have reinforced this position. ${ }^{11-16}$ Variation in the incidence estimates and the risk of recurrence of perioperative AF may contribute to the uncertainty in how to manage these patients.

The objective of this study was to systematically review the published literature to estimate: 1 ) the proportion of patients in whom new-onset AF is detected during hospitalization for non-cardiac surgery, and 2) the proportion of such patients in whom a recurrence of AF is detected over long-term followup. We also hypothesized that prospective studies with continuous ECG monitoring would have higher rates of AF detection than those that did not.

\section{Methods}

\section{Protocol}

The original review protocol was pre-registered (PROSPERO CRD42017068055; 1 September 2017). The differences between the original and final protocol are as follows. We initially planned to include observational and interventional studies including more than ten participants. Nevertheless, to make the number of eligible studies manageable, we changed this threshold to those with more than one hundred participants. As for the secondary outcomes, we initially planned to collect data on risk factors for the development of AF during hospitalization for non-cardiac surgery. To make the review manageable, and because these data are not readily combinable, we elected to forego collection of these data.

\section{Eligibility criteria}

This systematic review included prospective and retrospective observational studies of patients hospitalized for non-cardiac surgery. Included studies reported the incidence of new-onset AF during hospital admission for non-cardiac surgery, and/or the rate of $\mathrm{AF}$ recurrence following discharge from hospital in patients who had newonset perioperative AF. We only included studies with $>$ 100 participants. We excluded data from patients with a history of AF before hospital admission and from patients who underwent cardiac surgery.
Search strategy

We created the search strategy (available in the Electronic Supplementary Material [ESM] as eAppendix 1) with input from a medical librarian. We searched Ovid EMBASE (1974-November 2019), Ovid MEDLINE (1946-November 2019), and The Cochrane CENTRAL Library (Wiley) (November 2019). We did not impose language restrictions. The references of eligible papers were screened and experts were consulted to identify additional studies. We did not systematically hand-search conference abstracts or grey literature, but we did include conference abstracts that were captured by our database searches. Search results were imported into Covidence systematic review software (Veritas Health Innovation, Melbourne, Australia; available at www.covidence.org). Pairs of reviewers independently screened study titles and abstracts for eligibility using a pre-piloted template. If either reviewer thought the citation could be eligible, the full-text article was evaluated for eligibility. Full texts of the potentially eligible studies were retrieved and screened by pairs of reviewers. Reviewers recorded the principal reason for exclusion. Disagreements were resolved through discussion. Reviewers recorded the principal reason for exclusion.

\section{Data extraction and outcomes}

Independently, pairs of reviewers extracted data pertaining to study and participant characteristics, AF incidence, and $\mathrm{AF}$ recurrence into a pre-piloted database. Disagreements were resolved through discussion. Reviewers recorded type of surgery, methods for AF diagnosis, approach used to rule out a prior history of $\mathrm{AF}$, and whether studies used continuous ECG monitoring. Standard error (SE) for each study was calculated using the following formula: $S E=\sqrt{ }(($ new-onset $A F /$ total population) * (1- (new-onset AF/ total population))/total population). We grouped studies according to the major organ system under operation. We included studies that combined multiple types of surgeries under the heading "non-cardiac". Reviewers extracted the number of patients with $\mathrm{AF}$ and at risk, excluding those with a prior history of AF when this was reported. Reviewers contacted study authors to clarify ambiguities.

\section{Methodological quality of included studies}

Pairs of reviewers appraised the methodological quality of included studies using a pre-piloted tool based on the Newcastle-Ottawa Scale (Table 1). ${ }^{4,17}$ The three domains evaluated in each study were: exclusion of patients with a history of $\mathrm{AF}$, detection of $\mathrm{AF}$ in hospital, and detection of AF recurrence following the initial hospitalization. A score was allotted for each domain in each study. We considered 
TABLE 1 Quality assessment framework

\begin{tabular}{|c|c|c|c|}
\hline Domain & $\begin{array}{l}\text { Methods for exclusion of } \\
\text { a history of AF }\end{array}$ & $\begin{array}{l}\text { Methods for detection of } \\
\text { AF in hospital }\end{array}$ & $\begin{array}{l}\text { Methods for detection of } \\
\text { AF following hospital discharge }\end{array}$ \\
\hline \multirow{2}{*}{$\begin{array}{l}\text { Applicable } \\
\text { studies }\end{array}$} & $\begin{array}{l}\text { Incidence and } \\
\text { recurrence studies }\end{array}$ & $\begin{array}{l}\text { Incidence and } \\
\text { recurrence studies }\end{array}$ & Recurrence studies only \\
\hline & $\begin{array}{l}\text { Searched a prior source (e.g., } \\
\text { medical records) }\end{array}$ & $\begin{array}{l}\text { Continuous monitoring and } \\
\text { systematic ECG review }\end{array}$ & $\begin{array}{l}\text { Systematic ECG monitoring } \\
\text { protocol }\end{array}$ \\
\hline \multirow[t]{3}{*}{ Score } & Reported as no history of $\mathrm{AF}$ & $\begin{array}{l}\text { Routine ECGs or prospective, } \\
\text { continuous monitoring without } \\
\text { systematic review }\end{array}$ & $\begin{array}{l}\text { Systematic search for collected } \\
\text { ECGs }\end{array}$ \\
\hline & $\begin{array}{l}\text { Terms like "new-onset" are } \\
\text { used without meeting } \\
\text { selections } 1 \text { or } 2\end{array}$ & $\begin{array}{l}\text { Retrospective review of ECGs } \\
\text { collected non-systematically }\end{array}$ & $\begin{array}{l}\text { Systematic review of diagnoses or } \\
\text { non-systematic search for } \\
\text { collected ECGs }\end{array}$ \\
\hline & No description & No description & No description \\
\hline
\end{tabular}

$\mathrm{AF}=$ atrial fibrillation; $\mathrm{ECG}=$ electrocardiography.

studies to be of high quality if they searched medical records to exclude patients with a history of AF, used prospective, continuous, and systematic ECG review to detect $\mathrm{AF}$ in hospital, and employed a systematic monitoring protocol to detect $\mathrm{AF}$ recurrence.

Meta-analysis and subgroup tests

We used CMA software (Biostat Inc, Englewood, NJ, USA) to perform analyses. For each study, we inputed the incidence of perioperative $\mathrm{AF}$ as binomial proportions and calculated the accompanying standard errors. ${ }^{18}$ For the overall group of studies and for each type of surgery, we performed meta-analysis using a DerSimonian-Laird random effects model. ${ }^{19}$ We assessed between-study heterogeneity quantitatively with the $\mathrm{I}^{2}$ test and we planned to present a pooled estimate only if $\mathrm{I}^{2}<50 \%$. ${ }^{19} \mathrm{We}$ hypothesized that prospective studies with continuous ECG monitoring (i.e., quality score of 1 as defined in Table 1) would detect higher AF rates than those that did not, both in the overall population and in each different type of surgery. We tested this by creating a DerSimonian-Laird random effects model that pool studies based on quality score and compared subgroup effects with an interaction test. To estimate of the proportion of variance explained by continuous monitoring, we created a binary logistic meta-regression model and calculated a goodness-of-fit statistic and accompanying $\mathrm{R}^{2}$ analogue. ${ }^{20}$

\section{Results}

\section{Screening process}

The electronic search generated 39,233 unique citations (Fig. 1). After reference and full-text screening, 346 studies met eligibility criteria, including 345 that reported $\mathrm{AF}$ incidence and one that exclusively reported $\mathrm{AF}$ recurrence after hospital discharge. Twelve reported data on both incidence and recurrence.

Incidence studies

\section{Study Characteristics}

Three hundred and forty-five studies published between 1973 and 2019 reported the incidence of new-onset AF associated with non-cardiac surgery (Table 2; eAppendix 2, ESM). Five studies were published in Mandarin, four studies in Spanish, two studies in French, two studies in Japanese, and one study each in Hungarian, Russian, Icelandic, Romanian and Italian (eAppendix 3, ESM). The remaining studies were published in English. The intensive care unit (ICU) was the primary setting in 55 studies. Seventy studies were prospective cohort studies and 275 were retrospective. One hundred and fifty-three studies included patients who underwent pulmonary surgery, 32 studies included patients who underwent esophagectomy, 20 studies included patients who underwent thoracic surgery not otherwise specified, 60 studies included patients who underwent abdominal or digestive surgery, ten studies included patients who underwent vascular surgery, 26 studies included patients who underwent orthopedic surgery, and 44 studies reported aggregate data from patients who had undergone different types of non-cardiac surgery. The mean age of patients in the included studies ranged from 32 to $80 \mathrm{yr}$.

The methodologic quality of included studies varied greatly (eAppendix 2, ESM). One hundred and sixty-two studies $(47.0 \%)$ reported AF incidence after ruling out a 
Fig. 1 Study selection diagram (PRISMA Format)
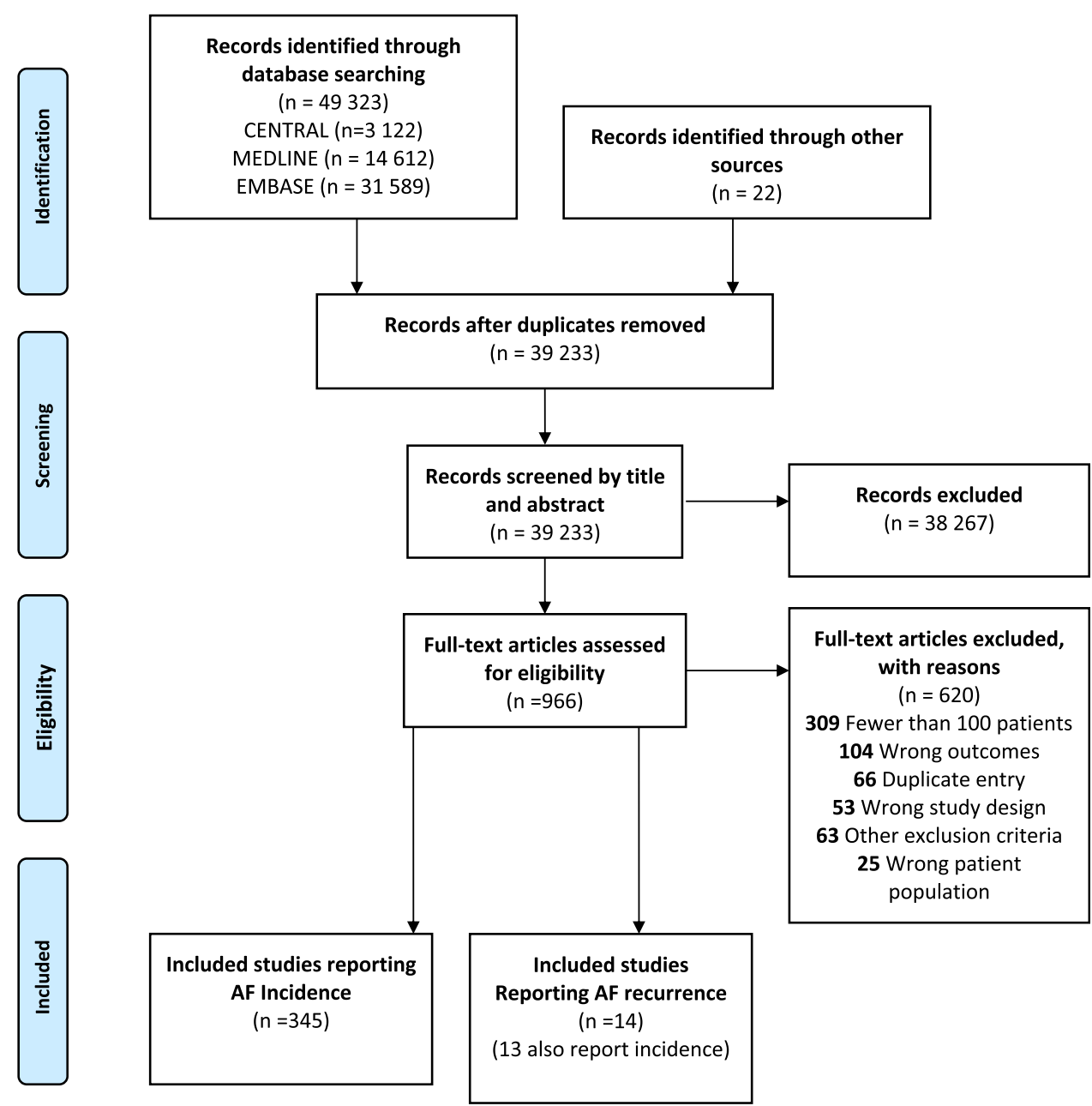

TABLE 2 Median incidence of new-onset atrial fibrillation according to type of surgery

\begin{tabular}{|c|c|c|c|c|c|c|}
\hline \multirow[t]{2}{*}{ Surgery type } & \multicolumn{3}{|c|}{$\begin{array}{l}\text { Continuous monitoring } \\
n=27 \text { Studies }\end{array}$} & \multicolumn{3}{|c|}{$\begin{array}{l}\text { No continuous monitoring } \\
n=318 \text { studies }\end{array}$} \\
\hline & $\begin{array}{l}\text { Number } \\
\text { of studies }\end{array}$ & $\begin{array}{l}\text { Total number } \\
\text { of patients }\end{array}$ & $\begin{array}{l}\text { Median (range) of } \mathrm{AF} \\
\text { incidence by study }(\%)\end{array}$ & $\begin{array}{l}\text { Number } \\
\text { of studies }\end{array}$ & $\begin{array}{l}\text { Total number } \\
\text { of patients }\end{array}$ & $\begin{array}{l}\text { Median (range) of AF } \\
\text { incidence by study (\%) }\end{array}$ \\
\hline $\begin{array}{l}\text { Pulmonary } \\
\text { resection/transplant }\end{array}$ & 15 & 3300 & $19.1(7.3-33.7)$ & 138 & 432,661 & $11.9(0.1-50.3)$ \\
\hline Esophagectomy & 2 & 242 & $13.9(7.8-20.0)$ & 30 & 13,421 & $13.4(1.1-27.7)$ \\
\hline Thoracic surgery NOS & 5 & 2280 & $12.5(8.7-25.2)$ & 15 & 19,706 & $16.0(1.1-29.0)$ \\
\hline Abdominal surgery & - & - & - & 60 & 194,498 & $3.3(0.004-16.9)$ \\
\hline Vascular surgery & 2 & 917 & $5.7(3.7-7.7)$ & 8 & 29,135 & $5.4(3.7-22.6)$ \\
\hline Orthopedic surgery & - & - & - & 26 & 585,613 & $3.0(0.1-9.3)$ \\
\hline Other & 3 & 1705 & $10.2(5.3-12.0)$ & 41 & $4,546,280$ & $3.6(0.3-17.2)$ \\
\hline Total & 27 & 8444 & $16.2(3.7-33.7)$ & 318 & $5,821,314$ & $8.1(0.004-50.3)$ \\
\hline
\end{tabular}

$\mathrm{AF}=$ atrial fibrillation; $\mathrm{NOS}=$ not otherwise specified

prior history of AF based on high-quality methods; 74 $(21.4 \%)$ described explicit searching of medical records to rule out a prior history of AF (quality score 1), and 88
$(25.5 \%)$ reported that patients in the study did not have a history of AF but did not mention searching medical records (quality score 2). Only 27 (7.8\%) studies monitored 
for $\mathrm{AF}$ using high-quality methods (prospective design with continuous ECG monitoring, quality score 1); five $(1.4 \%)$ of these studies used high-quality methods in both domains.

The incidence of new-onset $\mathrm{AF}$ during hospitalization for non-cardiac surgery reported in 345 studies $(n=$ $5,829,758)$ ranged from $<0.01 \%$ to $50.3 \%$. Table 2 reports the ranges of $\mathrm{AF}$ incidences according to type of surgery performed and whether continuous monitoring was used. Figure 2 summarizes the estimates of the incidence of newonset $\mathrm{AF}$ in studies that used continuous, prospective monitoring. Heterogeneity was substantial $\left(\mathrm{I}^{2}>90 \%\right)$ both overall and by surgical subgroup (eAppendices 4 and 5 , ESM); therefore, we did not pool results to generate a summary estimate. We tested the hypothesis that prospective studies with continuous ECG monitoring would find higher incidences of AF. To do this, we created a random effects binary logistic meta-regression model that compared the incidence of AF in studies with $\mathrm{AF}$ ascertainment quality scores of 1 with those with $\mathrm{AF}$ ascertainment quality scores of 2,3 , or 4 as defined in Table 1. Reported AF incidences were significantly higher in studies with continuous monitoring compared with those without (test of model $P<0.001$ ) according to the goodness-of-fit test $(P<0.001)$ and $R^{2}$ analogue 0.02 (eAppendix 5, ESM). Nevertheless, it suggested that only a small amount of variance (2\%) was explained by differences in monitoring intensity. We found no evidence of a subgroup effect when comparing studies using continuous monitoring with those that did not across surgical subtypes (eAppendix 4, ESM).

\section{Studies of long-term recurrence of AF}

Thirteen studies $(n=25,726)$ reported the long-term recurrence of $\mathrm{AF}$ following the index hospitalization (Table 3). Follow-up ranged from one month to 5.4 years after discharge. Four studies were prospective cohort studies and nine were retrospective. Follow-up duration and the methods used for AF detection varied markedly (Table 3). Only one study was assessed as having highquality methods for AF detection-Higuchi et al. ${ }^{21}$ used a systematic 12-month protocol for the surveillance of $\mathrm{AF}$ recurrence in cancer surgery patients with postoperative AF. Seventy-seven patients with confirmed postoperative AF wore either a two-week event-triggered recorder or a 24-hr Holter monitor at one month and 12 months postoperatively. Atrial fibrillation recurrence of at least

\section{Study name}

Pulmonary Surgery Amar D. 2000

Pulmonary Surgery Amar D. 2015

Pulmonary Surgery Barbetakis 2004

Pulmonary Surgery Cagirici U. 2005

Pulmonary Surgery Ciszewski P. 2013

Pulmonary Surgery Curtis J. 1998

Pulmonary Surgery Gomez-Caro A. 2006

Pulmonary Surgery Jesel L. 2017

Pulmonary Surgery Khalil M. 2013

Pulmonary Surgery Mita N. 2017

Pulmonary Surgery Nojiri 2010

Pulmonary Surgery Pu 2019

Pulmonary Surgery Riber 2014

Pulmonary Surgery Salvatici 2010

Pulmonary Surgery Tisdale 2009

Esophagectomy Hou JL. 2008

Esophagectomy Ojima 2017

Thoracic Surgery Cardinale D. 1999

Thoracic Surgery Cardinale D. 2007

Thoracic Surgery Cardinale D. 2016

Thoracic Surgery Salvatici 2014

Thoracic Surgery Xie 2018

Vascular Surgery Brathwaite 1998

Vascular Surgery Winkel 2010

Noncardiac Surgery Higuchi 2019

Noncardiac Surgery Seguin 2004

Noncardiac Surgery Xia 2014

Overall Incidence

\section{Statistics for each study}

\section{Event}

0.182

0.139

0.191

0.073

0.162

0.211

0.114

0.337

0.140

0.207

0.230

0.222

0.202

0.180

0.238

0.077

0.200

0.122

0.180

0.087

0.252

0.125

0.077

0.037

0.102

0.053

0.120

0.145

$\begin{array}{cc}\begin{array}{c}\text { Lower } \\ \text { limit }\end{array} & \begin{array}{c}\text { Upper } \\ \text { limit }\end{array} \\ 0.144 & 0.227 \\ 0.090 & 0.207 \\ 0.142 & 0.252 \\ 0.038 & 0.134 \\ 0.106 & 0.241 \\ 0.145 & 0.295 \\ 0.072 & 0.176 \\ 0.282 & 0.398 \\ 0.116 & 0.169 \\ 0.143 & 0.290 \\ 0.165 & 0.312 \\ 0.171 & 0.284 \\ 0.157 & 0.258 \\ 0.145 & 0.221 \\ 0.173 & 0.319 \\ 0.043 & 0.134 \\ 0.133 & 0.290 \\ 0.084 & 0.173 \\ 0.145 & 0.221 \\ 0.071 & 0.105 \\ 0.213 & 0.295 \\ 0.080 & 0.190 \\ 0.054 & 0.107 \\ 0.024 & 0.057 \\ 0.083 & 0.126 \\ 0.036 & 0.078 \\ 0.094 & 0.152 \\ 0.120 & 0.175\end{array}$

Event rate and $95 \% \mathrm{Cl}$



Fig. 2 Incidence of new-onset atrial fibrillation after non-cardiac surgery in prospective studies that used continuous electrocardiography. 


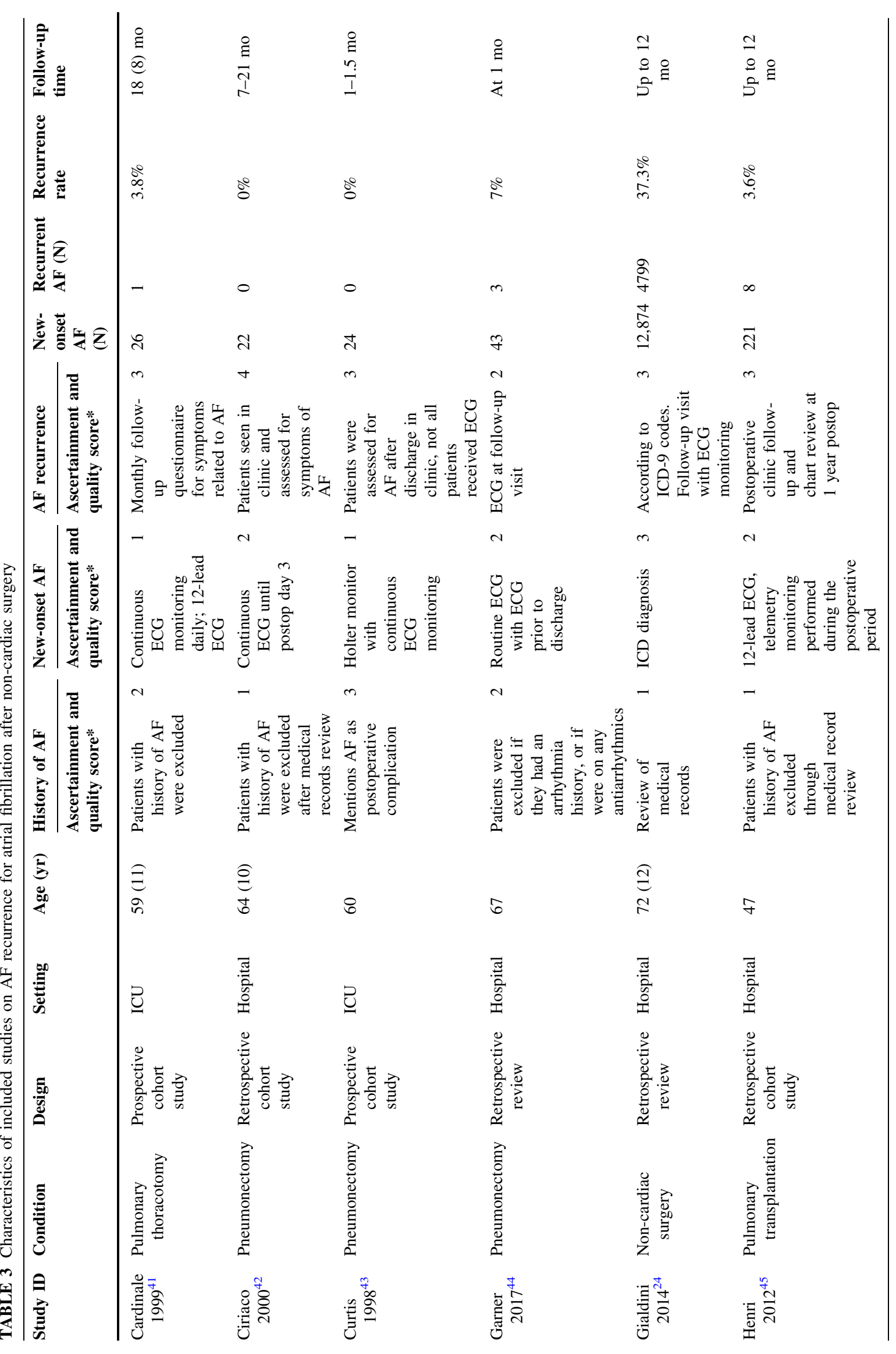




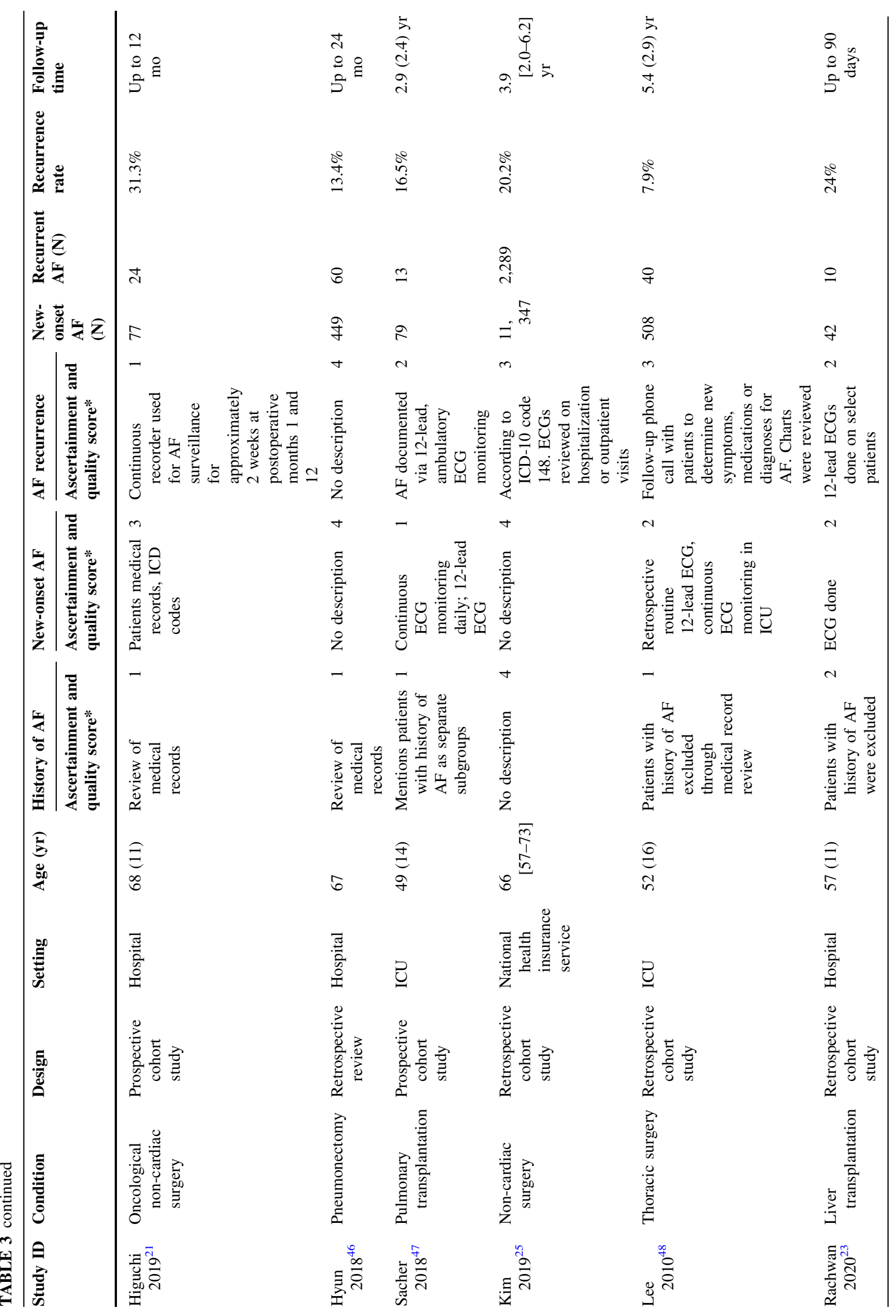


$30 \mathrm{sec}$, as confirmed by a cardiologist, was documented in $24(31.1 \%)$ patients. Four other studies reported AF recurrence rates of greater than $15 \%$. Jesel et al. followed patients with postoperative AF following lung transplantation for a mean (standard deviation) of 2.9 (2.4) years. $^{22}$ Patients were assessed weekly for three months, then monthly until one year, and every three months thereafter. Atrial fibrillation was documented by 12-lead ECG or ambulatory ECG monitoring, as reviewed by two cardiologists. The rate of AF recurrence was $16.5 \%$. Rachwan et al. followed patients who developed postoperative $\mathrm{AF}$ following liver transplantation. ${ }^{23}$ All were discharged from hospital in sinus rhythm. Of the 42 that had a 12-lead ECG in follow-up, ten (24\%) were in AF. Gialdini et al. used administrative claims data to identify Californian patients with new-onset perioperative $\mathrm{AF}^{24}$ Among 12,874 patients with no prior history of $\mathrm{AF}$ who developed new-onset perioperative AF, 4,799 (37.3\%) had another healthcare encounter for AF in the ensuing 12 months. Kim et al. used a similar approach with 11,347 patients in South Korea, finding that 2289 (20.2\%) had another encounter for AF over a median 47 months of follow-up. ${ }^{25}$

\section{Discussion}

In this systematic review, we found that among 345 published articles, the reported incidence of new-onset $\mathrm{AF}$ during hospitalization for non-cardiac surgery ranged from very rare $(<0.01 \%)$ to extremely common $(\sim 50 \%)$. This variability prohibited pooling data, even when reports of the same type of surgery were considered. Consistent with our hypothesis, prospective studies that used continuous ECG monitoring found higher incidences of AF than those that did not. Although some of the variability in study results was explained by detection methods and different surgical populations, much remains unexplained. There was similar variability in the 13 articles reporting on the long-term rate of AF recurrence in this population. Several studies, however, reported recurrence rates upwards of $20 \%$. Rigorously designed studies with continuous and prospective monitoring are required to more accurately estimate the incidence and long-term recurrence of newonset postoperative AF in patients who have undergone non-cardiac surgery.

To the best of our knowledge, this is the first systematic review to broadly assess the incidence and recurrence of new-onset perioperative AF following non-cardiac surgery. Most reviews to date have focused on cardiac surgery. ${ }^{26,27}$ Reviews in non-cardiac surgery have assessed surgical subpopulations and did not systematically assess study methodology for detection. ${ }^{28,29}$ This review has two 
important strengths. First, when possible, we excluded data from patients with a prior history of AF to isolate the population for whom the long-term prognosis is in question. Second, our methodological appraisal focused on methods used for AF detection and specifically tested the hypothesis that prospective studies with continuous ECG monitoring reported higher incidences of AF. As AF is often an intermittent arrhythmia, the probability of detecting AF increases with the intensity of ECG monitoring. ${ }^{30-32}$ As in our previous review of medical patients, studies with continuous monitoring found a significantly higher incidence of AF, although metaregression modelling suggested significant unexplained variance. ${ }^{33}$ These findings have informed the design of the AFOTS incidence study (clinicaltrials.org: NCT03552588) which is enrolling consecutive at-risk patients admitted to an ICU and continuously monitoring them with a continuous ECG monitor for up to 14 days. ${ }^{34}$

Accurate estimates of the incidence of new-onset perioperative AF are critical to guide future research in this patient population. There is interest in preventing newonset perioperative $\mathrm{AF}$ and in optimizing outcomes for affected patients. ${ }^{11-16}$ New-onset perioperative AF has been associated with adverse outcomes during the index hospital stay in at least two large, multi-centre studies. ${ }^{5,6}$ Nevertheless, the incidences of new-onset AF in these two studies $(2.5$ and $3.0 \%$ ) are at the lower end of what we found in this review. ${ }^{5,6}$ Confounding may explain these findings; AF may be noticed more frequently in patients who are more acutely ill with closer monitoring and in patients who stay longer with longer monitoring. In addition, worse outcomes for patients with AF could be explained by a higher burden of co-morbidities. ${ }^{35-37}$ This review underscores the need for standardized AF detection to minimize detection bias using prospective designs with continuous ECG monitoring. Continuous monitoring will also help estimate the minimum burden of AF that is associated with adverse outcomes.

Evidence is lacking to guide the long-term management of patients with new-onset perioperative AF. The majority of patients are discharged from hospital in sinus rhythm, whether it is due to spontaneous, pharmacologic, or electrical cardioversion. ${ }^{9,10}$ Nevertheless, clinicians are faced with the decision as to whether these patients should be treated for AF, with the most pressing question being whether to start them on long-term OAC treatment. Newonset perioperative $\mathrm{AF}$ has been associated with an increased long-term risk of stroke in large observational studies. $^{23,38,39}$ Nevertheless, the incidences of AF in these studies is again at the lower end of what was seen in our review $(0.4 \%, 0.8 \%, 2.2 \%)$, raising the question of selection bias.
We assessed long-term rates of AF recurrence in this population. This interest was driven by the hypothesis that patients who have a recurrence of AF following an initial presentation of AF perioperatively could be more likely to have paroxysmal $\mathrm{AF}$ and might therefore benefit from OAC. ${ }^{2,8,40}$ Nevertheless, we found very wide ranges in the long-term recurrence of AF. Only one 77-patient study systematically re-assessed patients using long-term ECG monitoring and found a cumulative recurrence rate of $31.1 \%$ over one year. ${ }^{21,40}$ Long-term ambulatory ECG monitoring may identify patients with recurrent $\mathrm{AF}$ who might benefit from $\mathrm{OAC}$ treatment as we await the results of randomized clinical trials. Ongoing studies are attempting to better define the long-term prognosis and management of this population. The AFOTS follow-up study attempts to overcome some of the limitations of prior studies. ${ }^{8}$ This study identifies patients with new-onset AF during hospitalization for medical illness or non-cardiac surgery who have returned to sinus rhythm at the time of hospital discharge. Cases and matched controls wear two 14-day Holter monitors over the ensuing 12 months. At least one randomized trial is being conducted in this population: Anticoagulation for Stroke Prevention In Patients With Recent Episodes of Perioperative Atrial Fibrillation After Noncardiac Surgery ASPIRE-AF is a pilot trial (clinicaltrials.org; NCT03968393) assessing the feasibility of a full study of OAC $v s$ no OAC in this population.

\section{Limitations of review}

This review is principally limited by the design and methodologic descriptions of the included studies. Most studies were either single centre or combined heterogeneous types of surgery. A small number of studies defined the minimum length of AF episodes they considered to be relevant. We did not collect data on the long-term risk of stroke associated with new-onset AF following non-cardiac surgery in this review. We are, however, assessing this in a separate, dedicated review (PROSPERO; CRD42017054309).

\section{Conclusions}

Rates of AF incidence and long-term recurrence of AF first detected following non-cardiac surgery vary markedly. Differences in the intensity of ECG monitoring and type of surgery may account for this variation. Atrial fibrillation recurs after hospital discharge in up to $37 \%$ of patients. 
Author contributions William F. McIntyre takes responsibility for the integrity of the data. William F. McIntyre, Jeff S. Healey, Richard P. Whitlock, and Emilie P. Belley-Côté contributed to study concept and design. William F. McIntyree, Maria E. Vadakken, Anand S. Rai, Terry Thach, Wajahat Syed, Kevin J. Um, Omar Ibrahim, Shreyash Dalmia, Akash Bhatnagar, Pablo A. Mendoza, Graham R. McClure, Toru Inami, Tianyi Zhang, and Jessica T. Huynh contributed to acquisition of data. William F. McIntyre, Emilie P. Belley-Côté, Maria E. Vadakken, Anand S. Rai, Richard P. Whitlock, Jeff S. Healey, Jessica Spence, Shrikant I. Bangdiwala, David Conen, and P.J. Devereaux contributed to analysis and interpretation of data. William F. McIntyre, Emilie P. Belley-Côté, and Maria E. Vadakken contributed to drafting of the manuscript. William F. McIntyre, Maria E. Vadakken, Anand S. Rai, and Shrikant I. Bangdiwala contributed to statistical analysis. Emilie P. Belley-Côté, Richard P. Whitlock, and Jeff $S$. Healey contributed to study supervision. All authors contributed to critical revision of the manuscript for important intellectual content.

\section{Disclosures None.}

Funding statement This study was supported by peer-reviewed research grants from the Canadian Stroke Prevention Intervention Network (C-SPIN) and the Canadian Cardiovascular Society-Bayer Vascular Awards Program. Dr McIntyre has fellowship Funding Awards from the C-SPIN, the McMaster Cooper Foundation and the Canadian Institutes of Health Research (CIHR). Dr. Spence has a graduate training award from the Canadian Institute of Health Research. Dr. Devereaux holds the McMaster University/ Hamilton Health Sciences Chair in Perioperative Care and a Tier 1 Canada Research Chair in Perioperative Medicine. Dr. Conen holds a McMaster University Department of Medicine Mid-Career Research Award. Dr. Healey is the Population Health Research Institute Chair in Cardiovascular Research. Dr. Whitlock holds a Canada Research Chair in Surgery.

Editorial responsibility This submission was handled by Dr. Hilary P. Grocott, former Editor-in-Chief, Canadian Journal of Anesthesia.

\section{References}

1. Lloyd-Jones DM, Wang TJ, Leip EP, et al. Lifetime risk for development of atrial fibrillation: the Framingham Heart Study. Circulation 2004; 110: 1042-6.

2. McIntyre WF, Connolly SJ, Healey JS. Atrial fibrillation occurring transiently with stress. Curr Opin Cardiol 2018; 33: $58-65$.

3. McIntyre WF, Um KJ, Alhazzani W, et al. Association of vasopressin plus catecholamine vasopressors versus catecholamines alone with atrial fibrillation in patients with distributive shock: a systematic review and meta-analysis. JAMA 2018; 319: 889-900.

4. McIntyre WF, Um KJ, Cheung CC, et al. Atrial fibrillation detected initially during acute medical illness: a systematic review. Eur Heart J Acute Cardiovasc Care 2019; 8: 130-41.

5. Bhave PD, Goldman LE, Vittinghoff E, Maselli J, Auerbach A. Incidence, predictors, and outcomes associated with postoperative atrial fibrillation after major noncardiac surgery. Am Heart J 2012; 164: 918-24.
6. Alonso-Coello P, Cook D, Xu SC, et al. Predictors, prognosis, and management of new clinically important atrial fibrillation after noncardiac surgery: a prospective cohort study. Anesth Analg 2017; 125: 162-9.

7. Brathwaite D, Weissman $C$. The new onset of atrial arrhythmias following major noncardiothoracic surgery is associated with increased mortality. Chest 1998; 114: 462-8.

8. McIntyre WF, Mendoza PA, Belley-Côté EP, et al. Design and rationale of the atrial fibrillation occurring transiently with stress (AFOTS) follow-up cohort study. Clin Cardiol 2018; 41: 1273-80.

9. Goldman L. Supraventricular tachyarrhythmias in hospitalized adults after surgery. Clinical correlates in patients over 40 years of age after major noncardiac surgery. Chest 1978; 73: 450-4.

10. Walsh SR, Tang T, Gaunt ME, Schneider HJ. New arrhythmias after non-cardiothoracic surgery. BMJ 2006; . https://doi.org/10. 1136/bmj.333.7571.715.

11. Gutierrez C, Blanchard DG. Diagnosis and treatment of atrial fibrillation. Am Fam Physician 2016; 94: 442-52.

12. Healey JS, Parkash R, Pollak T, Tsang T, Dorian P. Canadian Cardiovascular Society atrial fibrillation guidelines 2010: etiology and initial investigations. Can J Cardiol 2011; 27: 31-7.

13. January CT, Wann LS, Alpert JS, et al. 2014 AHA/ACC/HRS guideline for the management of patients with atrial fibrillation: a report of the American College of Cardiology/American Heart Association Task Force on Practice Guidelines and the Heart Rhythm Society. J Am Coll Cardiol 2014; 64: e1-76.

14. Fuster V, Rydén LE, Cannom DS, et al. 2011 ACCF/AHA/HRS focused updates incorporated into the ACC/AHA/ESC 2006 guidelines for the management of patients with atrial fibrillation: a report of the American College of Cardiology Foundation/ American Heart Association Task Force on practice guidelines. Circulation 2011; 123: e269-367.

15. Verma A, Cairns JA, Mitchell LB, et al. 2014 focused update of the Canadian Cardiovascular Society guidelines for the management of atrial fibrillation. Can J Cardiol 2014; 30: 1114-30.

16. Camm AJ, Lip GY, De Caterina R, et al. 2012 focused update of the ESC guidelines for the management of atrial fibrillation: an update of the 2010 ESC guidelines for the management of atrial fibrillation. Developed with the special contribution of the European Heart Rhythm Association. Eur Heart J 2012; 33: 2719-47.

17. Wells $G$, Shea B, O'Connell D, et al. The Newcastle-Ottawa Scale (NOS) for assessing the quality of nonrandomised studies in meta-analyses. Available from URL; http://www.ohri.ca/ programs/clinical_epidemiology/oxford.asp (accessed January 2021).

18. Hazra A, Gogtay N. Biostatistics series module 1: basics of biostatistics. Indian J Dermatol 2016; 61: 10-20.

19. Higgins JP, Green S. Cochrane Handbook for Systematic Reviews of Interventions Version 5.1.0: The Cochrane Collaboration; 2011.

20. Kelley GA, Kelley KS. Statistical models for meta-analysis: a brief tutorial. World J Methodol 2012; 2: 27-32.

21. Higuchi $S$, Kabeya $Y$, Matsushita $K$, et al. Perioperative atrial fibrillation in noncardiac surgeries for malignancies and one-year recurrence. Can J Cardiol 2019; 35: 1449-56.

22. Jesel L, Barraud J, Lim HS, et al. Early and late atrial arrhythmias after lung transplantation- incidence, predictive factors and impact on mortality. Circ J 2017; 81: 660-7.

23. Rachwan RJ, Kutkut I, Hathaway TJ, et al. Postoperative atrial fibrillation and flutter in liver transplantation: an important predictor of early and late morbidity and mortality. Liver Transpl 2020; 26 : 34-44. 
24. Gialdini $G$, Nearing $K$, Bhave $P D$, et al. Perioperative atrial fibrillation and the long-term risk of ischemic stroke. JAMA 2014; 312: 616-22.

25. Kim K, Yang PS, Jang E, et al. Long-term impact of newly diagnosed atrial fibrillation during critical care: a South Korean Nationwide cohort study. Chest 2019; 156: 518-28.

26. Lowres N, Mulcahy G, Jin K, Gallagher R, Neubeck L, Freedman $B$. Incidence of postoperative atrial fibrillation recurrence in patients discharged in sinus rhythm after cardiac surgery: a systematic review and meta-analysis. Interact Cardiovasc Thorac Surg 2018; 26: 504-11.

27. Maisel WH, Rawn JD, Stevenson WG. Atrial fibrillation after cardiac surgery. Ann Int Med 2001; 135: 1061-73.

28. Chebbout R, Heywood EG, Drake TM, et al. A systematic review of the incidence of and risk factors for postoperative atrial fibrillation following general surgery. Anaesthesia 2018; 73: 490-8.

29. Walsh SR, Tang T, Wijewardena C, Yarham SI, Boyle JR, Gaunt $M E$. Postoperative arrhythmias in general surgical patients. Ann R Coll Surg Engl 2007; 89: 91-5.

30. Chen $L Y$, Chung MK, Allen LA, et al. Atrial fibrillation burden: moving beyond atrial fibrillation as a binary entity: a scientific statement from the American Heart Association. Circulation 2018; 137: e623-44.

31. Gladstone DJ, Spring M, Dorian $P$, et al. Atrial fibrillation in patients with cryptogenic stroke. N Engl J Med 2014; 370: 2467-77.

32. McIntyre WF, Healey $J$. Stroke prevention for patients with atrial fibrillation: beyond the guidelines. J Atr Fibrillation 2017; . https://doi.org/10.4022/jafib.1475.

33. McIntyre WF, Cheung CC, Dingwall $O$, et al. Atrial fibrillation occurring transiently with stress during medical illness: a systematic review. Can J Cardiol 2016; . https://doi.org/10. 1016/j.cjca.2016.07.333

34. McIntyre WF, Lengyel AP, Healey JS, et al. Design and rationale of the atrial fibrillation occurring transiently with stress (AFOTS) incidence study. J Electrocardiol 2019; 57: 95-9.

35. Andrade J, Khairy P, Dobrev D, Nattel S. The clinical profile and pathophysiology of atrial fibrillation: relationships among clinical features, epidemiology, and mechanisms. Circ Res 2014; 114: 1453-68.

36. Higuchi $S$, Kabeya $Y$, Matsushita $K$, et al. Incidence and complications of perioperative atrial fibrillation after noncardiac surgery for malignancy. PLoS One 2019; . https://doi. org/10.1371/journal.pone.0216239.

37. Bessissow A, Khan J, Devereaux PJ, Alvarez-Garcia J, AlonsoCoello $P$. Postoperative atrial fibrillation in non-cardiac and cardiac surgery: an overview. J Thromb Haemost 2015; 13(Suppl 1): $\mathrm{S} 304-12$.
38. Butt JH, Olesen JB, Havers-Borgersen E, et al. Risk of thromboembolism associated with atrial fibrillation following noncardiac surgery. J Am Coll Cardiol 2018; 72: 2027-36.

39. Conen D, Alonso-Coello P, Douketis J, et al. Risk of stroke and other adverse outcomes in patients with perioperative atrial fibrillation 1 year after non-cardiac surgery. Eur Heart J 2020; 41: 645-51.

40. Higuchi S, Kabeya $Y$, Matsushita K, et al. The study protocol for PREDICT AF RECURRENCE: a PRospEctive cohort stuDy of surveIllanCe for perioperaTive Atrial Fibrillation RECURRENCE in major non-cardiac surgery for malignancy. BMC Cardiovasc Disord 2018; . https://doi.org/10.1186/s12872018-0862-9.

41. Cardinale D, Martinoni A, Cipolla CM, et al. Atrial fibrillation after operation for lung cancer: clinical and prognostic significance. Ann Thorac Surg 1999; 68: 1827-31.

42. Ciriaco P, Mazzone P, Canneto B, Zannini P. Supraventricular arrhythmia following lung resection for non-small cell lung cancer and its treatment with amiodarone. Eur J Cardiothorac Surg 2000; 18: 12-6.

43. Curtis JJ, Parker BM, McKenney CA, et al. Incidence and predictors of supraventricular dysrhythmias after pulmonary resection. Ann Thorac Surg 1998; 66: 1766-71.

44. Garner M, Routledge T, King JE, et al. New-onset atrial fibrillation after anatomic lung resection: predictive factors, treatment and follow-up in a UK thoracic centre. Interact Cardiovasc Thorac Surg 2017; 24: 260-4.

45. Henri $C$, Giraldeau $G$, Dorais $M$, et al. Atrial fibrillation after pulmonary transplantation: incidence, impact on mortality, treatment effectiveness, and risk factors. Circ Arrhythm Electrophysiol 2012; 5: 61-7.

46. Hyun J, Cho MS, Nam GB, et al. Natural course and implication of anticoagulation in patients with new-onset postoperative atrial fibrillation. Heart Rhythm 2018; 15 Suppl: S648-9 (abstract).

47. Sacher F, Jesel L, Borni-Duval C, et al. Cardiac rhythm disturbances in hemodialysis patients: early detection using an implantable loop recorder and correlation with biological and dialysis parameters. JACC Clin Electrophysiol 2018; 4: 397-408.

48. Lee $G, W u H$, Kalman $J M$, et al. Atrial fibrillation following lung transplantation: double but not single lung transplant is associated with long-term freedom from paroxysmal atrial fibrillation. Eur Heart J 2010; 31: 2774-82.

49. Turaga KK, Shah KU, Neill EO, Mittal SK. Does laparoscopic surgery decrease the risk of atrial fibrillation after foregut surgery? Surg Endosc 2009; 23: 204-8.

Publisher's Note Springer Nature remains neutral with regard to jurisdictional claims in published maps and institutional affiliations. 